ARTICLE

\title{
A high-conductivity $n$-type polymeric ink for printed electronics
}

Chi-Yuan Yang (10 1, Marc-Antoine Stoeckel (10 1, Tero-Petri Ruoko (1) 1, Han-Yan Wu' ${ }^{1}$ Xianjie Liu', Nagesh B. Kolhe ${ }^{2}$, Ziang Wu ${ }^{3}$, Yuttapoom Puttisong (1) 4, Chiara Musumeci', Matteo Massetti ${ }^{1}$, Hengda Sun (1) 1 , Kai Xu', Deyu Tu (1) 1, Weimin M. Chen (10 4, Han Young Woo (i) ${ }^{3}$, Mats Fahlman (1) ${ }^{1}$, Samson A. Jenekhe (1) ${ }^{2}$, Magnus Berggren (iD) $1,5,6$ \& Simone Fabiano (iD 1,5,6凶

Conducting polymers, such as the p-doped poly(3,4-ethylenedioxythiophene):poly(styrene sulfonate) (PEDOT:PSS), have enabled the development of an array of opto- and bioelectronics devices. However, to make these technologies truly pervasive, stable and easily processable, $n$-doped conducting polymers are also needed. Despite major efforts, no $n$-type equivalents to the benchmark PEDOT:PSS exist to date. Here, we report on the development of poly(benzimidazobenzophenanthroline):poly(ethyleneimine) (BBL:PEI) as an ethanolbased $n$-type conductive ink. BBL:PEl thin films yield an $n$-type electrical conductivity reaching $8 \mathrm{~S} \mathrm{~cm}^{-1}$, along with excellent thermal, ambient, and solvent stability. This printable $n$-type mixed ion-electron conductor has several technological implications for realizing highperformance organic electronic devices, as demonstrated for organic thermoelectric generators with record high power output and $n$-type organic electrochemical transistors with a unique depletion mode of operation. BBL:PEl inks hold promise for the development of nextgeneration bioelectronics and wearable devices, in particular targeting novel functionality, efficiency, and power performance.

\footnotetext{
${ }^{1}$ Laboratory of Organic Electronics, Department of Science and Technology, Linköping University, Norrköping, Sweden. ${ }^{2}$ Department of Chemical Engineering and Department of Chemistry, University of Washington, Seattle, WA, USA. ${ }^{3}$ Department of Chemistry, College of Science, Korea University, Seoul 136-713, Republic of Korea. ${ }^{4}$ Department of Physics, Chemistry and Biology, Linköping University, Linköping, Sweden. ${ }^{5}$ Wallenberg Wood Science Center, Linköping University, Norrköping, Sweden. ${ }^{6}$ n-Ink AB, 58330 Linköping, Sweden. ${ }^{凶}$ email: simone.fabiano@liu.se
} 
C onducting polymers are an enabling technology for numerous opto- and bioelectronics applications ${ }^{1}$. The versatile chemical synthesis, low-cost solution processability, and exclusive mechanical robustness endow conducting polymers with broad appeal in industries such as renewable energies, sensing, and healthcare ${ }^{2-4}$. A prototypical conducting polymer used in many opto- and bioelectronic devices is the poly(3,4-ethylenedioxythiophene) doped with poly(styrene sulfonate), PEDOT:PSS (Fig. 1a $)^{5}$. With $>100$ tons produced every year, PEDOT:PSS is the most successful hole-transporting ( $p$-type) conducting polymer. This mixed ion-electron conductor owes its success to a high electrical conductivity, spanning over several orders of magnitude and reaching values $>1000 \mathrm{~S} \mathrm{~cm}^{-1}$, an excellent ambient stability, and commercial availability as aqueous dispersions for processing via traditional coating and printing techniques ${ }^{6}$. Today, PEDOT: PSS has been employed as the charge extraction/injection layer in organic solar cells ${ }^{7,8}$ and light-emitting diodes ${ }^{9,10}$, as well as the active material in electrochromic displays ${ }^{11,12}$, actuators ${ }^{13,14}$, electrochemical transistors ${ }^{15-17}$, sensors ${ }^{18,19}$, supercapacitors ${ }^{20,21}$, stretchable electronics ${ }^{22,23}$, thermoelectrics ${ }^{24-26}$, and brain-inspired memories ${ }^{27,28}$. However, many opto- and bioelectronic devices and systems rely on the complementarity of both high-performance hole-transporting and electron-transporting ( $n$-type) materials.

Although several $n$-type conducting polymers can be doped to high conductivity $\left(>10 \mathrm{~S} \mathrm{~cm}^{-1}\right)$ after deposition, their applicability is severely restricted by the use of harmful halogenated solvents, lack of thermal, ambient, and solvent stability, as well as reliable solution processability, which often result in poor device performance (see Supplementary Table 1 for a literature survey of $n$-doped polymers). Various design and $n$-doping strategies, including planarization and stiffening of the polymer backbone ${ }^{29,30}$, engineering of the donor-acceptor character ${ }^{31,32}$, control of the molecular dopant counterion-polymer side-chain miscibility ${ }^{33,34}$, and use of all-polymer blends based on groundstate electron transfer ${ }^{35}$ are being explored. Despite great progress, $n$-doped conducting polymers do not yet meet the performance comparable to the best $p$-doped polymers ${ }^{36}$, so that no $n$-type equivalents to PEDOT:PSS currently exist.

Here we report an alcohol-based $n$-type conductive ink for printed electronics. The $n$-type ink is composed of the conjugated polymer poly(benzimidazobenzophenanthroline) $(\mathrm{BBL})^{37}$ doped with poly(ethyleneimine) (PEI $)^{38}$, an amine-based insulating polymer (Fig. 1b). The BBL:PEI polymer mixture is prepared via the formulation of an ethanol-based ink that is processable in air through simple spray-coating. After thermal activation, the $n$ type BBL:PEI thin films show an electrical conductivity as high as $8 \mathrm{~S} \mathrm{~cm}^{-1}$, as well as excellent thermal and ambient stability. We also find that the high conductivity can be retained even after washing the thin films with common organic solvents, which is particularly important for the development of multi-layered optoelectronic devices. We demonstrate the useability of this material as a printed active layer in thermoelectric generators (TEGs) with record-high power output $(56 \mathrm{nW}$ per $p-n$ pair at $\Delta T=50 \mathrm{~K})$. We further implement BBL:PEI as a mixed ionelectron conductor in organic electrochemical transistors (OECTs) and demonstrate $n$-type depletion mode of operation as well as new power-efficient logic devices when coupled to PEDOT:PSS-based OECTs. We anticipate that BBL:PEI inks will a
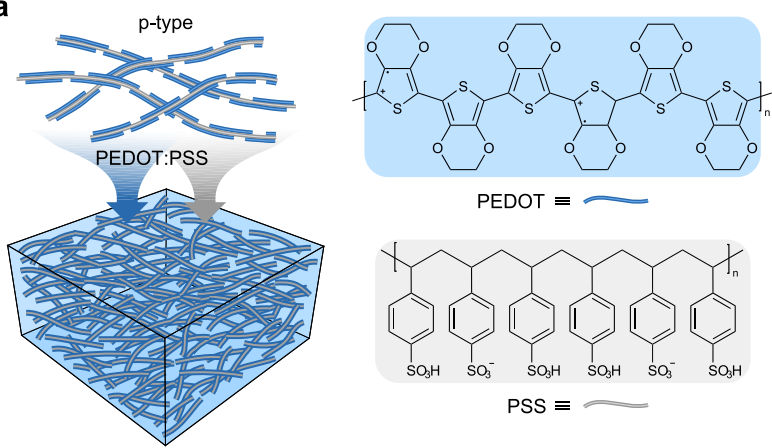

PEDOT $\equiv$

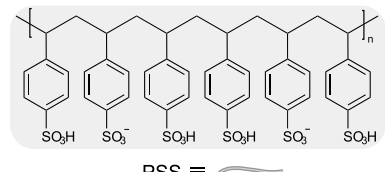

C
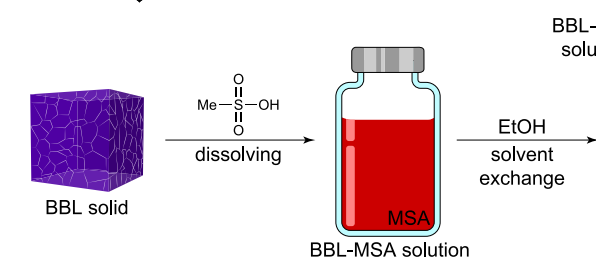

BBL-MSA solution

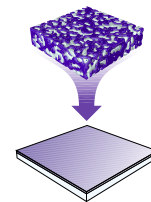

n-type conducting polyme b
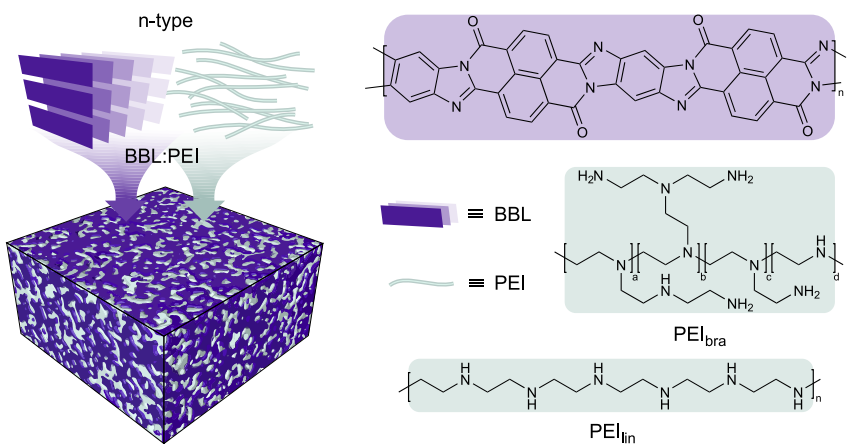

d

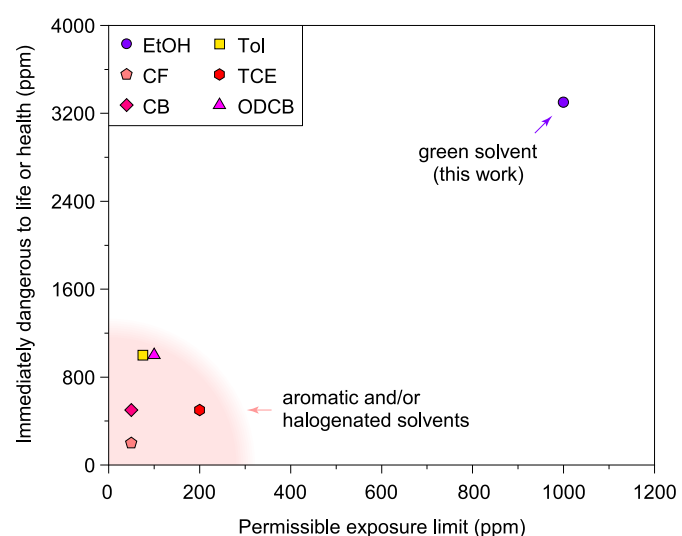

Fig. 1 Material processing. a Schematics of the film structure and chemical structure of $p$-type PEDOT:PSS. b Schematics of the film structure and chemical

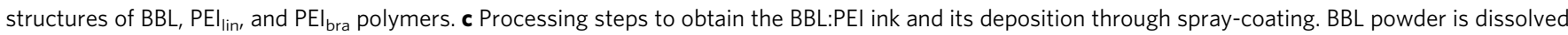
in MSA, followed by dispersion in ethanol through a solvent-exchange method, which results in the formation of BBL nanoparticles, and finally mixing with PEI to form the final BBL:PEI ink; BBL:PEl ink can be spray-coated in air, followed by thermal annealing, to form the highly conducting $n$-type film. $\mathbf{d}$ Health exposure limits of the solvent used for the BBL:PEI ink and comparison with other solvents typically used to process $n$-type conducting polymers (Tol, toluene; CF, chloroform; TCE, trichloroethylene; CB, chlorobenzene; and ODCB, 1,2-dichlorobenzene). Data from the US National Institute for Occupational Safety and Health (NIOSH, see Supplementary Table 2). 
have a similar impact on the field of organic electronics as the prototypical PEDOT:PSS.

\section{Results}

Ink formulation and processing. BBL:PEI ink was prepared by dissolving BBL in methanesulfonic acid (MSA), followed by solvent-exchange with ethanol under rapid stirring (Fig. 1c). This yields BBL nanoparticles with a size of about $20 \mathrm{~nm}$ in diameter (Supplementary Fig. 1). The BBL nanoparticles are then washed in ethanol and mixed with either linear PEI $\left(\mathrm{PEI}_{\text {lin }}\right)$ or branched $\mathrm{PEI}\left(\mathrm{PEI}_{\mathrm{bra}}\right)$ at different mass ratios, and sonicated to obtain the ethanol-based all-polymer conductive ink (see "Methods" for further details). The resulting ethanol-based ink is composed of BBL:PEI nanoparticles with a size of $30-100 \mathrm{~nm}$, depending on the PEI content (Supplementary Fig. 1). The ink can be processed in air and deposited over a large area by simple spray-coating technique. Because of the low boiling point of ethanol, BBL:PEI does not require thermal annealing for drying the films, whereas a thermal treatment under inert atmosphere or vacuum is needed to reach the high-conductivity values (vide infra). In addition, unlike the aromatic and halogenated solvents typically used to process $n$-doped conducting polymers, the use of green solvent such as ethanol is expected to facilitate the transition of BBL:PEI ink from lab to fab (Fig. 1d and Supplementary Table 2).

Film microstructure characterization. We carried out grazingincidence wide-angle X-ray scattering (GIWAXS) to determine the microstructure of the spray-coated BBL:PEI fin films (Fig. 2a-e and see Supplementary Figs. 2 and 3 for BBL:PEI $\mathrm{bra}_{\text {bra }}$ films). Pure BBL is primarily oriented edge-on on the substrate, exhibiting a strong lamellar $(100)$ peak at $q_{z}=0.794 \AA^{-1}(d$-spacing $=7.91 \AA)$ and a strong $\pi-\pi$ stacking (010) peak at $q_{x y}=1.846 \AA^{-1}(d$-spacing $=3.40$ $\AA)$. The diffraction pattern of pure $\mathrm{PEI}_{\text {lin }}$ shows very sharp rings that disappear when $\mathrm{PEI}_{\text {lin }}$ is blended with BBL, indicative of a good intermixing between the two polymeric phases. As the $\mathrm{PEI}_{\text {lin }}$ content increases, the BBL $\pi-\pi$ stacking distance decreases, reaching a value of $3.36 \AA$, which is indicative of a stronger $\pi-\pi$ interaction. This leads to higher $\pi-\pi$ stacking crystallinity with longer coherence lengths and lower paracrystalline disorder (Supplementary Figs. 4-6). At 50 wt\% $\mathrm{PEI}_{\text {lin }}$ content, two sets of lamellar diffraction peaks arise: (i) (100) diffraction peak with a packing distance of $7.23 \AA$, which is slightly smaller than that of pristine BBL, and (ii) (100)' diffraction peak with a packing distance of $11.7 \AA$, which is $3.79 \AA$ longer than that of pure BBL (Supplementary Figs. 7-9). This suggests that PEI chains intercalate between the BBL lamella without directly interfering with the BBL chain $\pi-\pi$ stacking. The addition of PEI results in a smoother film surface, as compared to pure BBL films (Fig. 2g, i). Conductive atomic force microscopy reveals the presence of con-

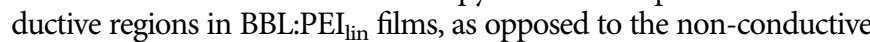
surface of pure BBL films (Fig. $2 \mathrm{~h}, \mathrm{j}$ and see Supplementary Fig. 10 for BBL:PEI bra films). X-ray photoelectron spectroscopy (XPS) reveals the presence of both negatively charged BBL and positively charged PEI chains (Fig. 2f and see also Supplementary Fig. 11 for a detailed $\mathrm{N}(1 s)$ spectra analysis).

Electrical performance. The electrical conductivity of BBL:PEI thin films is reported in Fig. 3a as a function of PEI content. Pure BBL films have an electrical conductivity as low as $10^{-5} \mathrm{~S} \mathrm{~cm}^{-1}$. When blended with $\mathrm{PEI}_{\text {lin }}$ and annealed, the electrical conductivity increases to $0.10 \pm 0.02 \mathrm{~S} \mathrm{~cm}^{-1}$ at $5 \mathrm{wt} \%$ PEI and saturates to $7.7 \pm 0.5 \mathrm{~S} \mathrm{~cm}^{-1}$ for $50 \mathrm{wt} \%$ PEI content. Because of the high density of electron-donating secondary amine groups in $\mathrm{PEI}^{39,40}$, a 5 min treatment at $150^{\circ} \mathrm{C}$ is enough to reach $1 \mathrm{~S} \mathrm{~cm}^{-1}$ $n$-type conductivity, whereas the maximum conductivity of $8 \mathrm{~S}$ $\mathrm{cm}^{-1}$ is obtained after $2 \mathrm{~h}$ of annealing (Supplementary Fig. 12). For comparison, we measured BBL films blended with $\mathrm{PEI}_{\text {bra }}$, bearing primary, secondary, and tertiary amines, and obtained a maximum conductivity of $4.0 \pm 0.1 \mathrm{~S} \mathrm{~cm}^{-1}$ for $50 \mathrm{wt} \%$ PEI content (Fig. 3a). When BBL is blended with polyethylenimine ethoxylated (PEIE), it reaches a conductivity of $1.4 \pm 0.1 \mathrm{~S} \mathrm{~cm}^{-1}$
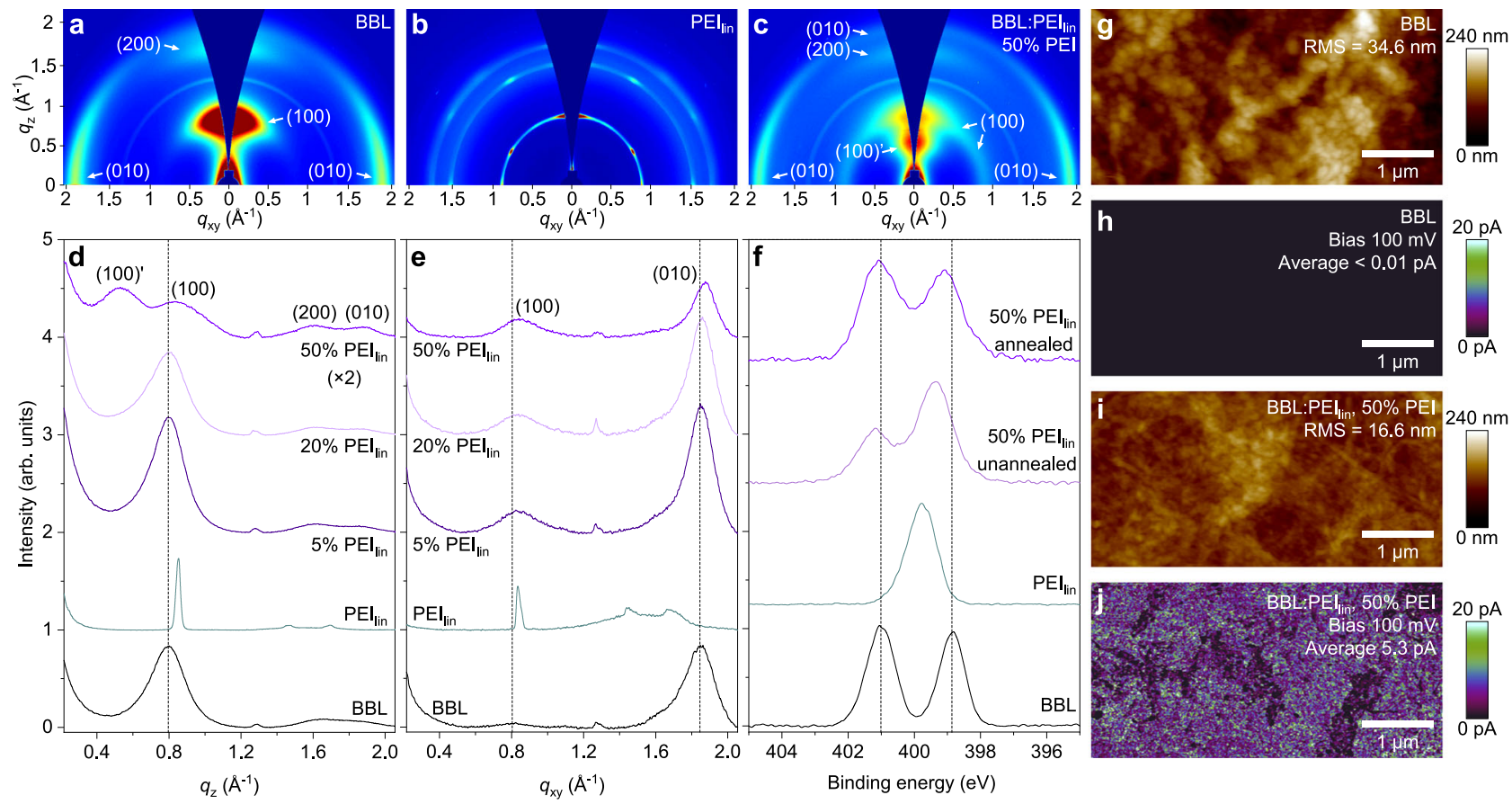

Fig. 2 Material characterization. a-c 2D GIWAXS patterns of BBL (a), PEl $\left.\right|_{\text {lin }}(\mathbf{b})$, and BBL:PEl $l_{\text {lin }}(50$ wt\% PEl, c) films. d, e Out-of-plane (d) and in-plane (e) GIWAXS line cuts of BBL, PEl lin, and BBL:PE| $\left.\right|_{\text {lin }}$ films. $\mathbf{f} \mathrm{N}(1 \mathrm{~s})$ XPS analysis of BBL, PEl lin, and BBL:PEl lin $(50$ wt\% PEI) films. g-j Atomic force microscope (AFM) images and corresponding conductive-AFM images of BBL ( $\mathbf{g}$ height image; $\mathbf{h}$ current image) and BBL:PEl lin (i height image; $\mathbf{j}$ current image). The root mean square (RMS) surface roughness and average current are also reported. 

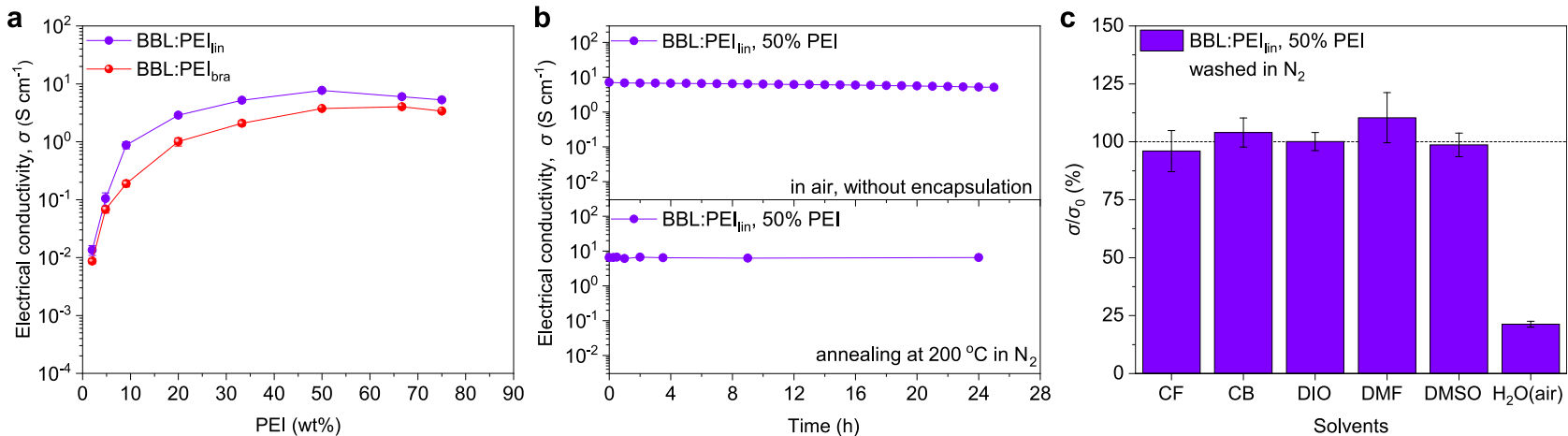

Fig. 3 Electrical properties and stability. a Conductivity of $B B L:\left.P E\right|_{\text {lin }}$ and $B B L:\left.P E\right|_{\text {bra }}$ for different PEl content. $\mathbf{b}$ Evolution of conductivity of BBL:PE| $\left.\right|_{\text {lin }}$ in air for $24 \mathrm{~h}$ and thermal stability when subjected to annealing at $200^{\circ} \mathrm{C}$ in nitrogen atmosphere over $24 \mathrm{~h}$. c Stability of BBL:PEl $l_{\text {lin }}$ exposed to common organic solvents (chloroform (CF), chlorobenzene (CB), 1,8-diiodooctane (DIO), dimethylformamide (DMF), and dimethylsulfoxide (DMSO)), showing changes in conductivity. Error bars indicate the SD of ten experimental replicates.

at BBL:PEIE 5:1, with higher PEIE content leading to a degradation of the electrical performance (Supplementary Fig. 13). Prior to thermal annealing, BBL:PEI films are stable for at least 2 days in air and do not suffer from any conductivity degradation (Supplementary Fig. 14). The conductivity is independent of film thickness $(d)$ for $d>50 \mathrm{~nm}$ (average $\sim 6 \mathrm{~S} \mathrm{~cm}^{-1}$ ), whereas it decreases to $\sim 1.5 \mathrm{~S} \mathrm{~cm}^{-1}$ when $d$ approaches the BBL nanoparticle size (i.e., for $20 \mathrm{~nm}$-thick samples, Supplementary Fig. 15). The out-of-plane electrical conductivity of BBL:PEI films is $0.1 \mathrm{~S} \mathrm{~cm}^{-1}$, which is less than two orders of magnitude lower than the in-plane conductivity (Supplementary Fig. 15). We interpret this anisotropic conductivity in terms of a percolating cluster model, developed for similar two-phase systems such as PEDOT:PSS ${ }^{41}$. The negatively charged BBL chains are preferentially ordered parallel to the substrate and are compensated by the long positively charged PEI chains, so that the latter are expected to have a preferential order parallel to the substrate as well. This anisotropy favors the in-plane conductivity as also observed in the case of PEDOT:PSS ${ }^{41}$. It is noteworthy that BBL: PEI forms an ohmic contact to various electrodes with work functions ranging from $5.1 \mathrm{eV}$ (PEDOT:PSS) to $2.8 \mathrm{eV}$ (Ca/Al) (Supplementary Fig. 16).

BBL:PEI shows excellent ambient stability, with the conductivity of $12 \mu \mathrm{m}$-thick films decreasing only about $20 \%$ upon $24 \mathrm{~h}$ exposure to air (Fig. 3b). This stability is induced by the combination of high work function $(4.19 \mathrm{eV}$, vide infra) and selfencapsulation in the micrometer-thick film, which inhibits the penetration of $\mathrm{H}_{2} \mathrm{O}$ and $\mathrm{O}_{2}$, as also observed for other recently reported $n$-doped polymers ${ }^{42}$. The electrical conductivity of thinner films $(\sim 100 \mathrm{~nm})$ drops to $0.1 \mathrm{~S} \mathrm{~cm}^{-1}$ after 10 days in air, whereas we observed a decrease of $<10 \%$ over 120 days when the thin films are stored in inert atmosphere (Supplementary Fig. 17). We also tested the thermal stability of BBL:PEI, which is crucial for applications that require continuous operation at high temperatures, such as solar cells or thermoelectrics. Remarkably, we did not observe any degradation of the conductivity (or Seebeck coefficient) even after annealing for $24 \mathrm{~h}$ at $200^{\circ} \mathrm{C}$ in inert atmosphere (see Fig. $3 \mathrm{~b}$ and Supplementary Fig. 18). Also, cycling the temperature between $20^{\circ} \mathrm{C}$ and $100^{\circ} \mathrm{C}$ did not induce any signs of degradation even after 10 cycles, whereas hightemperature annealing at $350^{\circ} \mathrm{C}$ decreased the conductivity of BBL:PEI $\mathrm{Pin}_{\text {lin }}$ by only $30 \%$ after $80 \mathrm{~min}$ (Supplementary Fig. 17).

We further tested the ability of BBL:PEI to maintain the highconductivity performance after washing the thin films with common organic solvents (Fig. 3c and Supplementary Table 3). This is particularly important for the development of multi-layer films that can be used in optoelectronic devices. BBL:PEI films can be washed with chloroform (CF), chlorobenzene (CB), 1,8-diiodooctane (DIO), dimethylformamide (DMF), and dimethylsulfoxide (DMSO) without affecting the conductivity, which is in striking contrast to other high-performance conducting polymers doped with small molecular dopants (Supplementary Fig. 19). We chose to test these solvents, as they are routinely used to process organic and hybrid active layers in solar cells and light-emitting diodes ${ }^{43,44}$. A more pronounced degradation of the conductivity is observed when BBL:PEI films are washed with water, with the conductivity decreasing by less than one order of magnitude to $1.5 \pm 0.1 \mathrm{~S} \mathrm{~cm}^{-1}$. These conductivity values are in line with those of a PEDOT:PSS formulation typically used in solar cells $\left(0.1-1 \mathrm{~S} \mathrm{~cm}^{-1}\right)^{45}$. The ambient, thermal, and solvent stability of BBL with $\mathrm{PEI}_{\text {bra }}$ is reported in Supplementary Fig. 20.

Spectroscopic characterization. To gain insight into the nature of charge carriers that contribute to transport in BBL:PEI, we performed ultraviolet photoelectron spectroscopy (UPS) and electron paramagnetic resonance (EPR) spectroscopy on BBL:PEI films with different PEI content (Fig. 4a and Supplementary Fig. 21). The work function of pure BBL is $4.32 \pm 0.05 \mathrm{eV}$ and shifts to $4.19 \pm 0.05 \mathrm{eV}$ when the PEI content increases to $50 \mathrm{wt} \%$. The EPR spectra corroborate the presence of polaronic species upon blending with PEI (Fig. 4a). The EPR spectra are reported in Supplementary Fig. 22 and the extracted data are presented in Supplementary Table 4. Although no EPR signal is detected for pure BBL, a strong EPR signal intensity is observed for BBL:PEI, reaching a spin density of $8 \times 10^{19} \mathrm{~cm}^{-3}$ for BBL:PEI with $50 \mathrm{wt} \%$ PEI content.

The ultraviolet-visible (UV-Vis) difference absorption spectra of BBL:PEI $\mathrm{P}_{\text {lin }}$ at various PEI content are reported in Fig. $4 \mathrm{~b}$. These spectra were obtained by subtracting the normalized absorption spectrum of BBL from the normalized BBL:PEI spectra presented in Supplementary Fig. 23, as PEI does not absorb in the reported wavelength range. Three polaronic absorption bands grow with increasing PEI content, with a sharp band at $400 \mathrm{~nm}$ and two overlapping wider bands centered around 725 and $890 \mathrm{~nm}$. This is consistent with previous $n$-doping ${ }^{30}$ and spectroelectrochemical studies ${ }^{46,47}$ of $\mathrm{BBL}$, corroborating the reduction of $\mathrm{BBL}$ chains in contact with PEI. The annealing of BBL:PEI increases the polaronic absorption drastically, showing that the polaron formation is thermally activated, although a weaker polaronic absorption is visible also for the unannealed blends (Supplementary Fig. 24). Polaron formation in BBL is also observed in Fourier-transformed infrared (FTIR) absorption spectra recorded on the same films, in which a broad polaronic absorption band, 

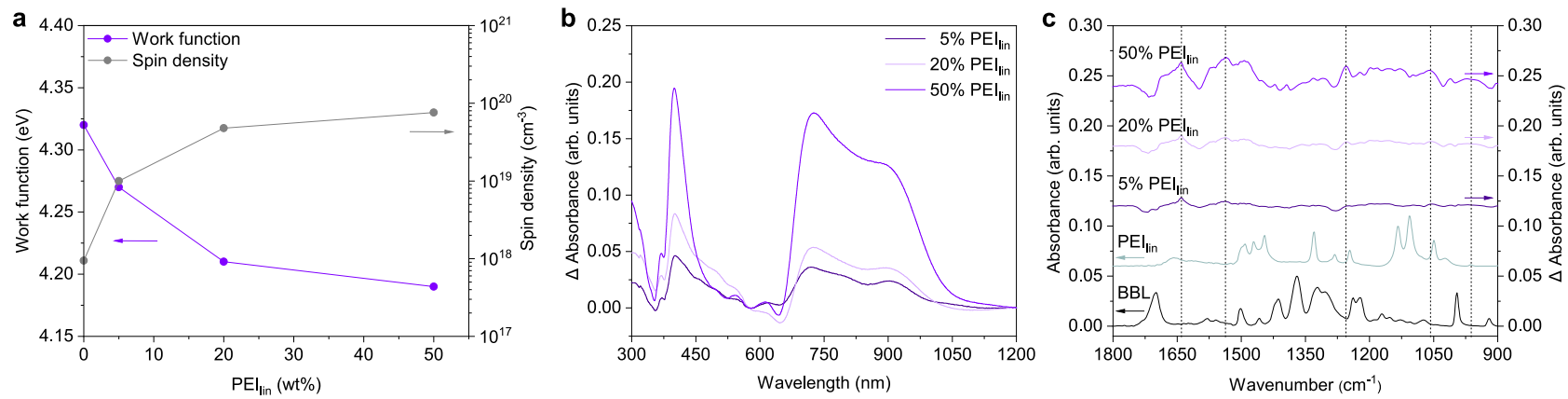

Fig. 4 Spectroscopic confirmation of doping. a Evolution of the work function measured by UPS and the spin density measured by EPR for BBL:PEl lin $_{\text {films }}$ with different PEI content. b Difference UV-Vis absorption spectra of BBL:PEI lin (50 wt\%) showing the evolution of polaron absorption induced by the increased PEI content. $\mathbf{c}$ The corresponding difference FTIR absorption spectra of the same samples. The difference absorption spectra in $\mathbf{b}$ and $\mathbf{c}$ were obtained by subtracting the BBL spectrum from the BBL:PEI lin spectra after normalization at $575 \mathrm{~nm}$ and $1370 \mathrm{~cm}^{-1}$ for UV-Vis and FTIR, respectively. A similar analysis is reported in Supplementary Fig. 26 for BBL:PEl ${ }_{\text {bra. }}$

with a maximum between 3500 and $3000 \mathrm{~cm}^{-1}$, appears upon increasing the PEI content (Supplementary Fig. 25). A similar absorption band has been previously reported for electrochemically doped BBL and is ascribed to an electronic transition in reduced $\mathrm{BBL}^{46}$. The difference FTIR absorption spectra in Fig. $4 \mathrm{c}$ highlight the emergence of five vibrational absorption bands induced by PEI that do not overlap with an existing vibration in either polymer; two strong polaronic peaks located at 1640 and $1535 \mathrm{~cm}^{-1}$, and three weaker peaks at 1255,1055 , and $970 \mathrm{~cm}^{-1}$. The band at $1640 \mathrm{~cm}^{-1}$, which is the fingerprint of negative polarons in $\mathrm{BBL}^{48}$, is assigned to antisymmetric $\mathrm{C}=\mathrm{O}$ stretching and coincides with a decrease in the intensity of the $\mathrm{C}=\mathrm{O}$ vibration in pristine $\mathrm{BBL}$ at $1700 \mathrm{~cm}^{-1}$. On the other hand, we assign the new polaronic band at $1535 \mathrm{~cm}^{-1}$ to $\mathrm{C}=\mathrm{C}$ vibrations in $\mathrm{BBL}$. Both the decrease of the $1700 \mathrm{~cm}^{-1}$ and the increase of the $1535 \mathrm{~cm}^{-1}$ bands are consistent with the formation of resonancestabilized quinone or hydroquinone structures due to the reduction of $\mathrm{BBL}^{47}$. FTIR measurements of unannealed and annealed BBL:PEI also prove that the polaron formation is mainly thermally activated, with the $\mathrm{C}=\mathrm{O}$ vibration of pristine $\mathrm{BBL}$ disappearing completely for annealed BBL:PEI (Supplementary Fig. 24). The three weaker vibrational peaks may further be associated with changes in BBL or reflect changes occurring in the $\mathrm{C}-\mathrm{N}$ vibration in PEI due to a positive charge residing in the amine. A vibrational change in PEI is most supported by the formation of the $1255 \mathrm{~cm}^{-1}$ peak, which lies close to the C-N stretching region of both aliphatic and aromatic amines. However, sharp polaronic absorption bands at 1255 and 1066 $\mathrm{cm}^{-1}$ have been previously reported for electrochemically reduced $\mathrm{BBL}$ as well ${ }^{46}$, indicating that assigning the weaker newly formed peaks here conclusively to changes in either BBL or PEI is not reasonable due to the overlap in the vibrational energies between the two polymers.

Organic TEGs. We then tested the thermoelectric properties of BBL:PEI films. The Seebeck coefficient decreases from $-482 \pm 1$ to $-65 \pm 1 \mu \mathrm{V} \mathrm{K}^{-1}$ with increasing PEI content (Fig. 5a and Supplementary Figs. 27 and 28). The negative sign of the Seebeck coefficient confirms the blend's $n$-type character. BBL:PEI presents a maximum power factor larger than $11 \mu \mathrm{W} \mathrm{m}{ }^{-1} \mathrm{~K}^{-2}$ with $33 \mathrm{wt} \% \mathrm{PEI}_{\text {lin }}$ content (Supplementary Fig. 27), which is remarkable for $n$-type polymers (Supplementary Table 1). We then demonstrated a flexible all-polymer TEG based on BBL:PEI (33 wt $\% \mathrm{PEI}_{\text {lin }}$ ) $n$-leg and PEDOT:PSS $p$-leg. The resulting TEG yields short-circuit currents and open-circuit voltages that are linearly proportional to the temperature gradient, with the TEG having an inner resistance of around $200 \Omega$ and a Seebeck coefficient of $131 \mu \mathrm{V} \mathrm{K}^{-1}$ (Supplementary Fig. 29). By connecting various load resistances (Fig. 5b and Supplementary Fig. 30), we measured a power output ranging from $0.54 \mathrm{nW}(\Delta T=5 \mathrm{~K})$ to $56 \mathrm{nW}(\Delta T=50 \mathrm{~K})$, which follows a square relationship with the temperature gradient. The power output per thermocouple is the highest reported value for in-plane all-polymer TEGs and much higher than organic TEGs with only a $p$-leg and metal connection (Fig. 5c and Supplementary Table 5). We also obtained similar results by printing silver electrodes (Supplementary Figs. 31 and 32), which enables the manufacturing of all-printed TEGs.

Organic electrochemical transistors. Finally, we tested BBL:PEI as an $n$-type organic mixed ionic-electronic conductor in OECTs. As BBL:PEI films are conductive in their pristine state, the resulting $n$-type OECT operates in the depletion mode. Figure $5 \mathrm{~d}$ shows the typical transfer characteristics of a BBL:PEI-based OECT (50 wt $\% \mathrm{PEI}_{\text {lin }}$, transfer curve cycling and output curves are shown in Supplementary Fig. 33). It is noteworthy that in analogy with PEDOT:PSS-based OECTs, we used BBL:PEI as both the channel and gate material. The source-drain current is high at zero gate voltage and decreases by three orders of magnitude when a negative voltage bias is applied to the gate. The maximum transconductance is $0.38 \mathrm{mS}$ at zero gate voltage. In addition, the device shows excellent cycling stability and fast response times $\left(\tau_{\text {on }}=167 \mathrm{~ms}\right.$ and $\tau_{\text {off }}=11 \mathrm{~ms}$, Supplementary Fig. 34). It is worth noting that $n$-type depletion-mode OECTs have not been realized before (see Fig. 5e and Supplementary Table 6 for a survey of the field) and their demonstration complements the current OECT technologies by bringing a new paradigm of logic circuitry. As an example, we paired an $n$-type BBL:PEI-based OECT to a $p$-type PEDOT:PSS-based OECT, both working in the depletion mode of operation, and demonstrated OECT-based ternary logic gates. This balanced ternary inverter can process three bits of information (“ +1 ," " 0 ”, and “- 1 "). The electrical characteristics of the $p$-type PEDOT:PSS-based OECTs are reported in Supplementary Fig. 33. Figure $5 f$ shows the ternary inverter layout and its voltage transfer characteristics, with a clear balanced ternary logic operation (Supplementary Fig. 35). During the two transition periods, the inverter exhibits similar gain values of up to 6 at $V_{\text {in }}=-0.07 \mathrm{~V}$ and $0.38 \mathrm{~V}$, while consuming $<10 \mu \mathrm{W}$. For comparison, unipolar binary inverters based on printed PEDOT:PSS OECTs consume about $1 \mathrm{~mW}^{17}$. The dashed line in Fig. $5 \mathrm{f}$ shows the simulated voltage transfer characteristics of the ternary inverter, which are in good agreement with the measured data (see "Methods" and Supplementary Fig. 36 for information regarding the SPICE model used for the simulation). It is noteworthy that our OECT-based ternary 

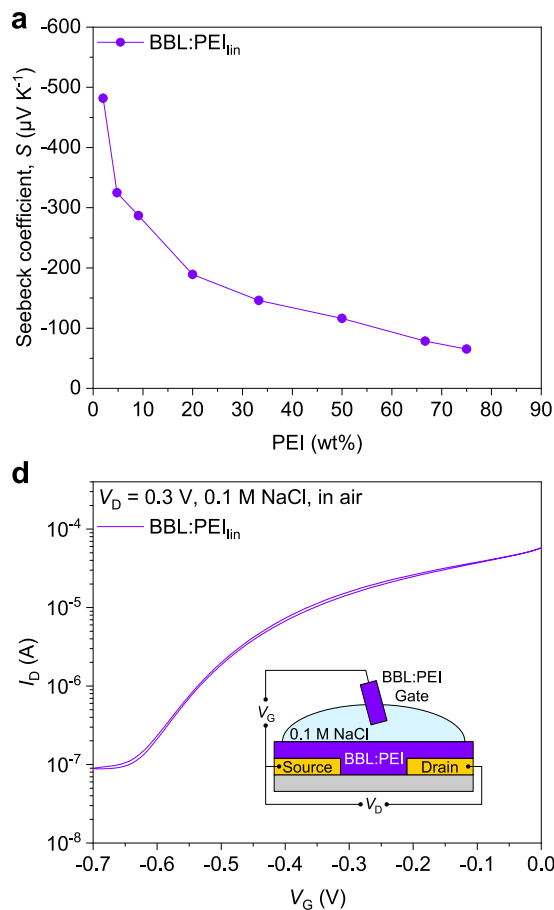
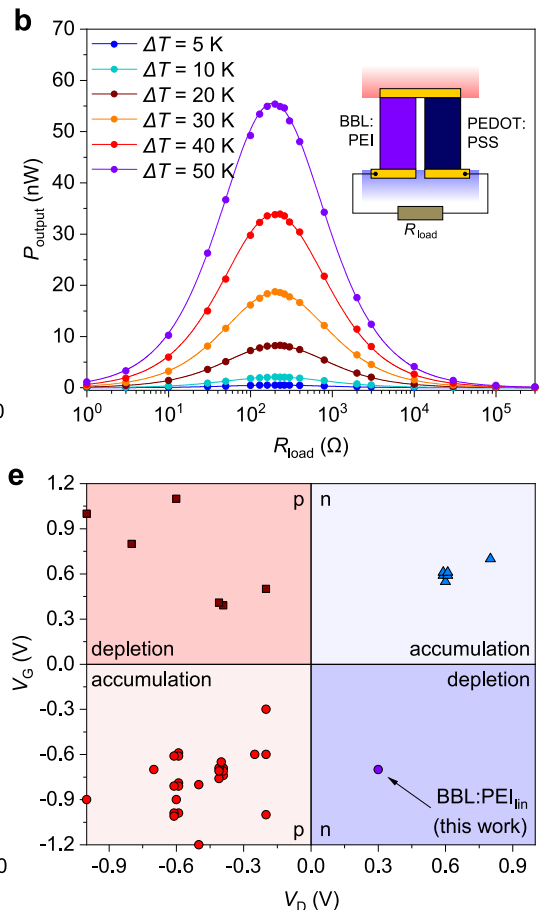
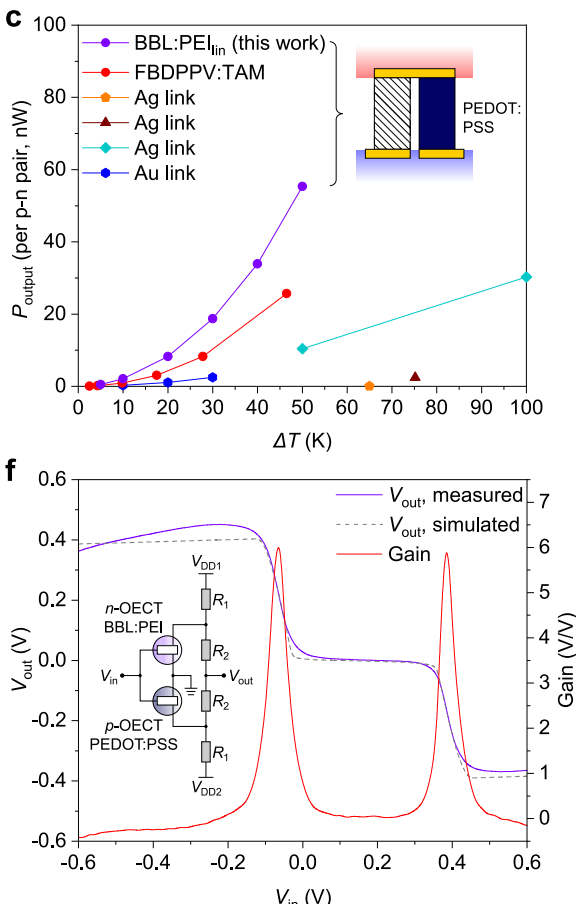

Fig. 5 Applications. a Seebeck coefficient of BBL:PEI lin. b Power output vs. resistance loads recorded at different temperature gradients for a planar thermoelectric module composed of a PEDOT:PSS $p$-type leg and a BBL:PEl $n$-type leg. c Power output per $p$ - $n$ pair vs. temperature gradient in this work, compared with other planar thermoelectric modules presented in literature. $\mathbf{d}$ Transfer curve of BBL:PEI-based OECT measured in air with $0.1 \mathrm{M} \mathrm{NaCl}$ electrolyte, demonstrating $n$-type conduction behavior in depletion mode. e Gate voltage-drain voltage map of the different regimes observed for organic semiconductor-based OECTs with both types of conduction from the literature (Supplementary Table 6). $\mathbf{f}$ Voltage output, gain, and simulated behavior of the ternary inverter integrating a PEDOT:PSS OECT for the $p$-side and BBL:PEI OECT for the $n$-side. Error bars indicate the SD of ten experimental replicates.

inverters outperform similar silicon-based tunneling ternary inverters in terms of voltage gain (Supplementary Fig. 37) ${ }^{49}$.

\section{Discussion}

In summary, we developed a high-conductivity ethanol-based $n$ type conductive ink with striking performance and stability, which holds great promise for printed electronics, energy technology, and bioelectronics. The negative charges on the conjugated polymer BBL are compensated by the positive charges on the amine-based insulating polymer PEI, resulting in an $n$-doped polymer-polymer ink that is processable in air through simple spray-coating. BBL:PEI thin films show an electrical conductivity as high as $8 \mathrm{~S} \mathrm{~cm}^{-1}$, as well as excellent thermal, ambient, and solvent stability. We demonstrated the application of this material as the active layer in printed TEGs, exhibiting record high power output ( $56 \mathrm{nW}$ per $p-n$ pair at $\Delta T=50 \mathrm{~K}$ ) when combined with the equivalent $p$-type counterpart PEDOT:PSS. We also explored the mixed ion-electron conductor properties of BBL:PEI in OECTs and demonstrated $n$-type depletion mode of operation as well as power-efficient logic devices when coupled to PEDOT: PSS-based OECTs. We anticipate that the $n$-type BBL:PEI will have a similar impact on the field of organic electronics as the prototypical $p$-type PEDOT:PSS and will offer solutions to currently unsolved problems where complementary hole and electron transport is required, with tremendous potential impact in next-generation organic opto- and bioelectronic devices.

\section{Methods}

Materials. BBL was synthesized following a procedure reported previously ${ }^{50}$. In brief, polyphosphoric acid $(250 \mathrm{~g})$ was added to a $500 \mathrm{~mL}$ three-necked flask fitted with an overhead stirrer and nitrogen inlet/outlet. The polyphosphoric acid was deoxygenated by heating overnight at $110^{\circ} \mathrm{C}$ with nitrogen bubbling through the stirred acid. Then, $1.92 \mathrm{~g}(6.75 \mathrm{mmol})$ of 1,2,4,5-tetraaminobenzene tetrahydrochloride was added, under a nitrogen atmosphere at $50^{\circ} \mathrm{C}$. The mixture was heated overnight at $75^{\circ} \mathrm{C}$ and $1,4,5,8$-naphthalenetetracarboxylic dianhydride $(1.81 \mathrm{~g}, 6.75 \mathrm{mmol})$ was then added. The mixture was slowly heated $\left(4^{\circ} \mathrm{C} \mathrm{min}^{-1}\right)$ to $180^{\circ} \mathrm{C}$ and maintained at that temperature for $10 \mathrm{~h}$. The resulting viscous solution was poured out of the flask at $180^{\circ} \mathrm{C}$ into a beaker and allowed to cool to room temperature. The polymer was precipitated in methanol, using a blender to facilitate mixing. The fibrous brown material was washed twice with methanol and water, and dried at $200{ }^{\circ} \mathrm{C}$ under reduced pressure $(0.40 \mathrm{~mm} \mathrm{Hg})$. Precipitation from $500 \mathrm{~g}$ of MSA and drying in the above manner gave BBL as dark purple fibers with metallic luster $\left(2.15 \mathrm{~g}, 95 \%\right.$ yield, $\eta=11.6 \mathrm{dL} \mathrm{g}^{-1}$ in $\mathrm{MSA}$ at $30^{\circ} \mathrm{C}, M_{\mathrm{w}}=60.5$ $\mathrm{kDa}) . \mathrm{PEI}_{\text {lin }}\left(M_{\mathrm{n}}=2.5 \mathrm{kDa}\right.$, polydispersity index $\left.(\mathrm{PDI})<1.3\right), \mathrm{PEI}_{\mathrm{bra}}\left(M_{\mathrm{n}}=10 \mathrm{kDa}\right.$, $\mathrm{PDI}=1.5)$, MSA, and ethanol were purchased from Sigma-Aldrich and were used as received. PEDOT:PSS (Clevios PH1000) was purchased from Heraeus Holding $\mathrm{GmbH}$.

Sample preparation. The BBL:PEI ink was fabricated through a surfactant-free method. BBL $(150 \mathrm{mg})$ was dissolved in MSA $(75 \mathrm{~mL})$ to form a deep red solution, then the BBL-MSA solution was added dropwise to ethanol $(300 \mathrm{~mL})$ under highspeed stirring (1500 r.p.m.). During the solvent-exchange, dark purple BBL nanoparticles were generated. The BBL nanoparticles were collected by centrifugation $(1700 \times g, 30 \mathrm{~min})$ and washed with ethanol for six times until neutral. The neutral BBL nanoparticles were re-dispersed in ethanol to obtain a dispersion (about $1 \mathrm{mg} \mathrm{mL}^{-1}$ ). PEI was then added to the BBL nanoparticle dispersion and the mixture was further homogenized in ultrasonic bath for $1 \mathrm{~h}$ to form the final BBL:PEI ink. The BBL:PEI ink can be further diluted for casting thin films in various thickness. BBL:PEI thin films were fabricated by spray-casting in air, by means of a standard HD-130 air-brush $(0.3 \mathrm{~mm})$ with atomization air pressure of 2 bar. After spray-casting, the BBL:PEI thin films were annealed at $140{ }^{\circ} \mathrm{C}$ for $2 \mathrm{~h}$ inside a nitrogen-filled glovebox or under vacuum to get the conducting film.

UPS and XPS spectroscopy. XPS experiment was carried out in a Scienta ESCA 200 system with a base pressure of $2 \times 10^{-10}$ mbar equipped with an SES 200 electron analyzer, a monochromatic $\mathrm{Al} \mathrm{Ka} \mathrm{X-ray} \mathrm{source}(1486.6 \mathrm{eV})$, and a helium discharge lamp (21.22 eV) for XPS and UPS, respectively. All spectra were collected at normal emission and were calibrated by a sputter-cleaned $\mathrm{Au}$ film with the Fermi level at $0 \mathrm{eV}$ and the $\mathrm{Au}(4 f)$ peak at $84.0 \mathrm{eV}$. The work function was extracted from the edge of the secondary electron cutoff in UPS while applying 
$\mathrm{a}-3 \mathrm{~V}$ bias on the sample. To extract the underlying mechanism of the charge transfer between $\mathrm{PEI}_{\mathrm{lin}}$ and BBL, i.e., negatively charged $\mathrm{BBL}$ and positively charged $\mathrm{PEI}_{\mathrm{lin}}$ in the mixed sample, the XPS peak of $\mathrm{N}(1 s)$ was deconvoluted. The $\mathrm{N}(1 s)$ spectral features obtained from fitting of pristine $\mathrm{PEI}_{\text {lin }}$ and BBL films were used in the deconvolution of the blend samples, with additional features added corresponding to charged species created upon mixing $\mathrm{PEI}_{\text {lin }}$ and $\mathrm{BBL}$.

Electron paramagnetic resonance. Quantitative EPR experiments were performed at the Swedish Interdisciplinary Magnetic Resonance Centre at Linköping University, using a Bruker Elexsys E500 spectrometer operating at about $9.8 \mathrm{GHz}$ (X-band). EPR spectra were recorded in dark at room temperature.

AFM and c-AFM microscopy. Atomic force microscopy (AFM) and conductiveAFM (c-AFM) were performed in a Dimension 3100/Nanoscope IV system, equipped with a c-AFM module (current sensitivity $1 \mathrm{nA} \mathrm{V}^{-1}$ ) from Bruker. Soft $\mathrm{Pt} / \mathrm{Cr}$-coated silicon probes $\left(k=0.2 \mathrm{~N} \mathrm{~m}^{-1}\right)$ were used to simultaneously map topography and current in contact mode at a constant load force of $2-5 \mathrm{nN}$. The current maps were obtained by constantly biasing the Au substrate, while keeping the scanning AFM probe at ground. All the measurements were performed at room temperature in ambient atmosphere.

Grazing-incidence wide-angle X-ray scattering. GIWAXS experiments were performed at Beamline 9A at the Pohang Accelerator Laboratory in South Korea. The X-ray energy was $11.07 \mathrm{eV}$ and the incidence angle was $0.12^{\circ}$. Samples were measured in vacuum and total exposure time was $10 \mathrm{~s}$. The scattered X-rays were recorded by a charge-coupled device detector located $221.7788 \mathrm{~mm}$ from the sample. All samples for GIWAXS measurements had similar thickness of around $100 \mathrm{~nm}$.

UV-Vis-near infrared and FTIR. BBL, PEI, and BBL:PEI films were prepared on calcium fluoride windows following the above-mentioned procedure for optical characterization. All measurements were performed with the film inside an airtight sample holder, which was sealed in a nitrogen-filled glovebox. UV-Vis-near infrared absorption spectra of the films were measured with Perkin Elmer Lambda 900 with a resolution of $2 \mathrm{~nm}$. The FTIR spectra were measured in transmission mode with Bruker Equinox 55, averaging 200 scans with a resolution of $4 \mathrm{~cm}^{-1}$ and zerofilling factor of 2 .

Electrical characterization. Electrical conductivity and Seebeck coefficient measurements were performed inside a nitrogen-filled glovebox using a Keithley 4200SCS semiconductor characterization system. Five nanometers of chromium as an adhesive layer and $50 \mathrm{~nm}$ of gold were thermally evaporated on cleaned glass substrates through a shadow mask, forming electrodes with a channel length/width of $30 \mu \mathrm{m} / 1000 \mu \mathrm{m}$ for the electrical and $0.5 \mathrm{~mm} / 15 \mathrm{~mm}$ for Seebeck coefficient characterizations. Four-probe conductivity measurements were also performed and showed comparable resistances to the two-probe measurements (Supplementary Fig. 38).

Thermoelectric generators. The TEGs had an in-plane geometry with one $p / n$-leg pair module prepared on a $25 \mu \mathrm{m}$-thick polyethylene naphthalate (PEN) substrate. For the $p$-leg, we used PEDOT:PSS (PH1000) treated with DMSO (5 wt\%). Considering the different electrical conductivity of secondary-doped PEDOT:PSS and BBL:PEI, the widths of the $p / n$ legs were set to $2.5 \mathrm{~mm} / 20 \mathrm{~mm}$, respectively; the leg lengths and thicknesses were both $2.5 \mathrm{~mm}$ and $10 \mu \mathrm{m}$, respectively. First, the chromium/gold $(5 \mathrm{~nm} / 50 \mathrm{~nm})$ electrodes were evaporated on the PEN substrate through a shadow mask. Then, PEDOT:PSS and BBL:PEI legs were printed through spraycoating the respective dispersions in air. The samples were then annealed in nitrogen at $140^{\circ} \mathrm{C}$ and was followed by encapsulation with CYTOP. For TEGs with silver electrodes, the PEDOT:PSS and BBL:PEI legs were directly printed on PEN substrate in air and the silver paste was printed on the top of legs to form the electrodes. The samples were then annealed and encapsulated using the same method.

OECTs and ternary inverters. OECTs had a lateral-gate geometry. OECTs were fabricated on glass substrates (standard microscope glass). The substrates were washed by acetone, water, and isopropanol sequentially in ultrasonic bath and dried by nitrogen. Then chromium/gold $(5 \mathrm{~nm} / 50 \mathrm{~nm})$ were deposited on the substrates through shadow mask to form the source/drain electrodes with channel length $L=30 \mu \mathrm{m}$ and channel width $W=1 \mathrm{~mm}$. For $n$-type depletion mode OECT, a $50 \mathrm{~nm}$-thick BBL:PEI channel and gate layer were spray-coated through shadow mask with gate size of $5 \mathrm{~mm} \times 5 \mathrm{~mm}$. The samples were annealed in nitrogen at $140^{\circ} \mathrm{C}$ for $2 \mathrm{~h}$ and, finally, a protection tape insulating layer were added. For the $p$-type depletion mode OECT, PEDOT:PSS (containing $1 \mathrm{wt} \%$ of (3-glycidyloxypropyl)trimethoxysilane ${ }^{51}$ and $5 \mathrm{wt} \%$ of ethylene glycol) was homogenized in ultrasonic bath for $30 \mathrm{~min}$ and spin-coated at 4000 r.p.m. on the substrate. The PEDOT:PSS layer were patterned by protection tape to form the channel and gate (gate size of $5 \mathrm{~mm} \times 5 \mathrm{~mm}$ ). The samples were annealed at $120^{\circ} \mathrm{C}$ in air for $1 \mathrm{~min}$ and dipped into $\mathrm{TAM}^{52}$ ethanol solution $\left(5-20 \mathrm{mg} \mathrm{mL}^{-1}\right)$ for $1 \mathrm{~min}$. After annealing in nitrogen at $140^{\circ} \mathrm{C}$ for $60 \mathrm{~min}$, the samples were finally insulated by using a protection tape. For ternary inverter, one $n$-type
OECT, one $p$-type OECT, and four resistors $\left(R_{1}=820 \mathrm{k} \Omega\right.$ and $R_{2}=330 \mathrm{k} \Omega$, Fig. 5e) were integrated through silver paste lines. The $n$-type, $p$-type OECTs, and ternary inverters were tested in air with $0.1 \mathrm{M} \mathrm{NaCl}$ aqueous electrolyte.

SPICE model. SPICE models of the depletion-mode $n$-/p-type OECTs are developed in B2 SPICE (EMAG Technologies, Inc.). Both $n$-type and $p$-type models are built to simulate the voltage transfer characteristics of the ternary inverter. The model parameters in the sub-circuits (resistors, capacitors, and diodes) are the same for both types of OECTs, except for the polarity and threshold voltage of the depletion-mode transistors.

\section{Data availability}

The authors declare that the main data supporting the findings of this study are available within the paper and its Supplementary Information files. Source data are provided with this paper.

Received: 24 November 2020; Accepted: 16 March 2021; Published online: 21 April 2021

\section{References}

1. Guo, X. \& Facchetti, A. The journey of conducting polymers from discovery to application. Nat. Mater. 19, 922-928 (2020).

2. Kosco, J. et al. Enhanced photocatalytic hydrogen evolution from organic semiconductor heterojunction nanoparticles. Nat. Mater. 19, 559-565 (2020).

3. Ohayon, D. et al. Biofuel powered glucose detection in bodily fluids with an $\mathrm{n}$ type conjugated polymer. Nat. Mater. 19, 456-463 (2020).

4. Someya, T., Bao, Z. \& Malliaras, G. G. The rise of plastic bioelectronics. Nature 540, 379-385 (2016).

5. Groenendaal, L., Jonas, F., Freitag, D., Pielartzik, H. \& Reynolds, J. R. Poly(3,4ethylenedioxythiophene) and its derivatives: past, present, and future. Adv. Mater. 12, 481-494 (2000).

6. Elschner, A., Kirchmeyer, S., Lovenich, W., Merker, U. \& Reuter, K. PEDOT: Principles and Applications of an Intrinsically Conductive Polymer (CRC, 2010).

7. Chen, H.-Y. et al. Polymer solar cells with enhanced open-circuit voltage and efficiency. Nat. Photonics 3, 649-653 (2009).

8. Xia, Y., Sun, K. \& Ouyang, J. Solution-processed metallic conducting polymer films as transparent electrode of optoelectronic devices. Adv. Mater. 24, 2436-2440 (2012)

9. Tan, Z. K. et al. Bright light-emitting diodes based on organometal halide perovskite. Nat. Nanotechnol. 9, 687-692 (2014).

10. Huang, F., Wu, H. \& Cao, Y. Water/alcohol soluble conjugated polymers as highly efficient electron transporting/injection layer in optoelectronic devices. Chem. Soc. Rev. 39, 2500-2521 (2010).

11. Andersson, P., Forchheimer, R., Tehrani, P. \& Berggren, M. Printable allorganic electrochromic active-matrix displays. Adv. Funct. Mater. 17, 3074-3082 (2007)

12. Lang, A. W., Österholm, A. M. \& Reynolds, J. R. Paper-based electrochromic devices enabled by nanocellulose-coated substrates. Adv. Funct. Mater. 29, 1903487 (2019).

13. Hamedi, M. M. et al. Electrically activated paper actuators. Adv. Funct. Mater 26, 2446-2453 (2016).

14. Taccola, S. et al. Toward a new generation of electrically controllable hygromorphic soft actuators. Adv. Mater. 27, 1668-1675 (2015).

15. Rivnay, J. et al. Organic electrochemical transistors. Nat. Rev. Mater. 3, 17086 (2018).

16. Cea, C. et al. Enhancement-mode ion-based transistor as a comprehensive interface and real-time processing unit for in vivo electrophysiology. Nat. Mater. 19, 679-686 (2020).

17. Andersson Ersman, P. et al. All-printed large-scale integrated circuits based on organic electrochemical transistors. Nat. Commun. 10, 5053 (2019).

18. Cicoira, F. et al. Influence of device geometry on sensor characteristics of planar organic electrochemical transistors. Adv. Mater. 22, 1012-1016 (2010).

19. Nikolou, M. \& Malliaras, G. G. Applications of poly(3,4-ethylenedioxythiophene) doped with poly(styrene sulfonic acid) transistors in chemical and biological sensors. Chem. Rec. 8, 13-22 (2008).

20. Zhang, C. et al. Highly flexible and transparent solid-state supercapacitors based on RuO2/PEDOT:PSS conductive ultrathin films. Nano Energy 28 495-505 (2016)

21. Manjakkal, L., Pullanchiyodan, A., Yogeswaran, N., Hosseini, E. S. \& Dahiya, R. A Wearable supercapacitor based on conductive PEDOT:PSS-coated cloth and a sweat electrolyte. Adv. Mater. 32, 1907254 (2020).

22. Wang, Y. et al. A highly stretchable, transparent, and conductive polymer. $\mathrm{Sci}$. Adv. 3, e1602076 (2017). 
23. Kayser, L. V. \& Lipomi, D. J. Stretchable conductive polymers and composites based on PEDOT and PEDOT:PSS. Adv. Mater. 31, 1806133 (2019).

24. Coates, N. E. et al. Effect of interfacial properties on polymer-nanocrystal thermoelectric transport. Adv. Mater. 25, 1629-1633 (2013).

25. $\mathrm{Xu}, \mathrm{S}$. et al. High-performance PEDOT:PSS flexible thermoelectric materials and their devices by triple post-treatments. Chem. Mater. 31, 5238-5244 (2019).

26. Fan, Z. \& Ouyang, J. Thermoelectric properties of PEDOT:PSS. Adv. Electron. Mater. 5, 1800769 (2019).

27. Gkoupidenis, P., Schaefer, N., Garlan, B. \& Malliaras, G. G. Neuromorphic functions in PEDOT:PSS organic electrochemical transistors. Adv. Mater. 27, 7176-7180 (2015)

28. van de Burgt, Y. et al. A non-volatile organic electrochemical device as a lowvoltage artificial synapse for neuromorphic computing. Nat. Mater. 16, 414-418 (2017).

29. Lu, Y. et al. Rigid Coplanar polymers for atable n-type polymer thermoelectrics. Angew. Chem. Int. Ed. 58, 11390-11394 (2019).

30. Wang, S. et al. Thermoelectric properties of solution-processed n-doped ladder-type conducting polymers. Adv. Mater. 28, 10764-10771 (2016).

31. Yang, C.-Y. et al. Enhancing the n-type conductivity and thermoelectric performance of donor-acceptor copolymers through donor engineering. $A d v$. Mater. 30, 1802850 (2018).

32. Wang, S. et al. A chemically doped naphthalenediimide-bithiazole polymer for n-type organic thermoelectrics. Adv. Mater. 30, 1801898 (2018).

33. Liu, J. et al. Enhancing molecular n-type doping of donor-acceptor copolymers by tailoring side chains. Adv. Mater. 30, 1704630 (2018).

34. Kiefer, D. et al. Double doping of conjugated polymers with monomer molecular dopants. Nat. Mater. 18, 149-155 (2019).

35. $\mathrm{Xu}, \mathrm{K}$. et al. Ground-state electron transfer in all-polymer donor-acceptor heterojunctions. Nat. Mater. 19, 738-744 (2020).

36. Sun, H., Guo, X. \& Facchetti, A. High-performance n-type polymer semiconductors: applications, recent development, and challenges. Chem $\mathbf{6}$, 1310-1326 (2020)

37. Babel, A. \& Jenekhe, S. A. High electron mobility in ladder polymer field-effect transistors. J. Am. Chem. Soc. 125, 13656-13657 (2003).

38. Zhou, Y. et al. A universal method to produce low-work function electrodes for organic electronics. Science 336, 327-332 (2012).

39. Fabiano, $\mathrm{S}$. et al. Poly(ethylene imine) impurities induce $\mathrm{n}$-doping reaction in organic (semi)conductors. Adv. Mater. 26, 6000-6006 (2014).

40. Seidel, K. F. et al. Single-step formation of a low work function cathode interlayer and n-type bulk doping from semiconducting polymer/ polyethylenimine blend solution. ACS Appl. Mater. Interfaces 12, 28801-28807 (2020).

41. van de Ruit, K. et al. The curious out-of-plane conductivity of PEDOT:PSS Adv. Funct. Mater. 23, 5787-5793 (2013).

42. Han, J. et al. Dichlorinated dithienylethene-based copolymers for air-stable ntype conductivity and thermoelectricity. Adv. Funct. Mater. 30, 2005901 (2020).

43. Zhang, S., Ye, L., Zhang, H. \& Hou, J. Green-solvent-processable organic solar cells. Mater. Today 19, 533-543 (2016).

44. McDowell, C., Abdelsamie, M., Toney, M. F. \& Bazan, G. C. Solvent additives: key morphology-directing agents for solution-processed organic solar cells. Adv. Mater. 30, 1707114 (2018).

45. Zhang, S. et al. Poly(3,4-ethylenedioxythiophene):polystyrene sulfonate films with low conductivity and low acidity through a treatment of their solutions with probe ultrasonication and their application as hole transport layer in polymer solar cells and perovskite solar cells. Org. Electron. 32, 149-156 (2016).

46. Yohannes, T. et al. Multiple electrochemical doping-induced insulator-toConductor transitions observed in the conjugated ladder polymer polybenzimidazobenzophenanthroline (BBL). J. Phys. Chem. B 104, 9430-9437 (2000).

47. Wilbourn, K. \& Murray, R. W. The electrochemical doping reactions of the conducting ladder polymer benzimidazobenzophenanthroline (BBL). Macromolecules 21, 89-96 (1988).

48. Fazzi, D., Fabiano, S., Ruoko, T.-P., Meerholz, K. \& Negri, F. Polarons in $\pi$ conjugated ladder-type polymers: a broken symmetry density functional description. J. Mater. Chem. C. 7, 12876-12885 (2019).

49. Jeong, J. W. et al. Tunnelling-based ternary metal-oxide-semiconductor technology. Nat. Electron. 2, 307-312 (2019).

50. Arnold, F. E. \& Deusen, R. L. V. Preparation and properties of high molecular weight, soluble oxobenz[de]imidazobenzimidazoisoquinoline ladder polymer. Macromolecules 2, 497-502 (1969).

51. Håkansson, A. et al. Effect of (3-glycidyloxypropyl)trimethoxysilane (GOPS) on the electrical properties of PEDOT:PSS films. J. Polym. Sci. B Polym. Phys. 55, 814-820 (2017).
52. Yang, C.-Y. et al. A thermally activated and highly miscible dopant for $\mathrm{n}$-type organic thermoelectrics. Nat. Commun. 11, 3292 (2020).

\section{Acknowledgements}

We thank Duyen K. Tran (U. Washington) for helpful discussion and Qilun Zhang (Linköping U.) for assistance with the dynamic light scattering measurements. This work was financially supported by the Knut and Alice Wallenberg foundation, the Swedish Research Council (2016-03979 and 2020-03243), ÅForsk (18-313 and 19-310), Olle Engkvists Stiftelse (204-0256), VINNOVA (2020-05223), and the Swedish Government Strategic Research Area in Materials Science on Functional Materials at Linköping University (Faculty Grant SFO-Mat-LiU 2009-00971). H.Y. Woo acknowledges the financial support from the National Research Foundation of Korea (NRF2020M3H4A3081814 and 2019R1A6A1A11044070). Work at the University of Washington was supported by the National Science Foundation (DMR-2003518). We acknowledge MAX IV Laboratory for time on Beamline SPECIES-APXPS under Proposal 20200356. Research conducted at MAX IV, a Swedish national user facility, is supported by the Swedish Research council under contract 2018-07152, the Swedish Governmental Agency for Innovation Systems under contract 2018-04969, and Formas under contract 2019-02496.

\section{Author contributions}

S.F. conceived and designed the experiments. C.-Y.Y. developed the BBL:PEI ink. C.-Y.Y., M.-A.S., H.-Y. Wu, and K.X. performed the electrical characterization measurements and analyzed the data. T.-P.R. recorded and analyzed the UV-Vis-NIR and FTIR data. C.-Y.Y., N.B.K., and S.A.J. synthesized BBL. Z.W., C.-Y.Y., and H.Y. Woo measured and analyzed the GIWAXS data. H.S. helped with the ink production. C.M. recorded and analyzed the AFM and c-AFM data. X.L. and M.F. recorded and analyzed the UPS and XPS spectra. Y. P. and W.M.C. performed and analyzed the EPR data. C.-Y.Y. and M.M. fabricated and tested the thermoelectric generator. C.-Y.Y. and D.T. fabricated and tested the OECTs and ternary logic inverters. D.T. designed the ternary inverter and performed SPICE model simulation. C.-Y.Y., M.-A.S., T.-P.R., M.B., and S.F. wrote the manuscript. All authors contributed to discussion and manuscript preparation.

\section{Funding}

Open access funding provided by Linköping University.

\section{Competing interests}

C.-Y.Y., M.-A.S., M.B., and S.F. filed two provisional patent applications related to this work (applications numbers PCT/EP2020/082815 and PCT/EP2020/082821, filed 20 November 2020), and founded $\mathrm{n}$-Ink AB. The other authors declare no competing interests.

\section{Additional information}

Supplementary information The online version contains supplementary material available at https://doi.org/10.1038/s41467-021-22528-y.

Correspondence and requests for materials should be addressed to S.F.

Peer review information Nature Communications thanks Jian Pei and the other, anonymous, reviewer(s) for their contribution to the peer review of this work. Peer reviewer reports are available.

Reprints and permission information is available at http://www.nature.com/reprints

Publisher's note Springer Nature remains neutral with regard to jurisdictional claims in published maps and institutional affiliations.

\section{(i)}

Open Access This article is licensed under a Creative Commons Attribution 4.0 International License, which permits use, sharing, adaptation, distribution and reproduction in any medium or format, as long as you give appropriate credit to the original author(s) and the source, provide a link to the Creative Commons license, and indicate if changes were made. The images or other third party material in this article are included in the article's Creative Commons license, unless indicated otherwise in a credit line to the material. If material is not included in the article's Creative Commons license and your intended use is not permitted by statutory regulation or exceeds the permitted use, you will need to obtain permission directly from the copyright holder. To view a copy of this license, visit http://creativecommons.org/ licenses/by/4.0/.

(C) The Author(s) 2021, corrected publication 2022 